\title{
Gene expression profiles of the three major salivary glands in rats
}

\author{
Tomoko NASHIDA ${ }^{1}$, Ritsuko $\mathrm{SATO}^{2}$, Akane Imai $^{1}$, and Hiromi ShimomURA ${ }^{1}$ \\ ${ }^{1}$ Department of Biochemistry, Nippon Dental University, School of Life Dentistry at Niigata, and ${ }^{2}$ Department of Dental Hygiene, \\ Nippon Dental University College at Niigata, Japan
}

(Received 9 August 2010; and accepted 28 September 2010)

\begin{abstract}
The protein components of saliva reflect the condition of the whole body as well as the salivary glands. The aim of this study is to characterize the gene expression profiles in each of the rat major salivary glands - the parotid, submandibular, and sublingual glands. Gene expression was analyzed using DNA microarrays, and observed differences in expression of representative genes were confirmed by quantitative, real-time polymerase chain reaction. Among the glands, the contribution to the high expression of genes encoding various proteins, specifically mucin 10 , proline-rich glycoproteins, proline-rich protein 2, proline-rich proteoglycans, cystatin 10, amylase, deoxyribonuclease I, and von Ebner's gland protein, was significantly greater in the parotid gland than the other glands. The submandibular and sublingual glands had similar gene expression profiles that differed from profile of the parotid gland. For example, the genes encoding mucin 19 and ovomacroglobulin were highly expressed only in the submandibular and sublingual glands. In summary, we characterized gene expression in the rat major salivary glands and provided basic information on salivary gland marker proteins.
\end{abstract}

The parotid, submandibular, and sublingual glands are the three major salivary glands in rodents and humans, and secretions from these glands are mixed in the oral cavity and contribute $26 \%, 69 \%$, and $5 \%$, respectively, to the contents of whole saliva in humans (29). Recently, analyses of saliva components have been used to evaluate manifestation of some diseases $(5,26,34)$. As the collection of saliva is safe and easy, it is useful as a diagnostic in the early detection and evaluation of diseases. For example, in the saliva from patients with Sjögren's disease, levels of pro-inflammatory cytokines, lactoferrin, and antibody against $\alpha$-fodrin are higher than the levels in healthy individuals $(5,26,34)$.

Additionally, salivary flow rate is an indicator of

Address correspondence to: Dr. Tomoko Nashida, Department of Biochemistry, Nippon Dental University, School of Life Dentistry at Niigata, 1-8 Hamaura-cho, Chuo-ku, Niigata 951-8580, Japan

Tel: +81-25-267-1500 ext 592, Fax: +81-25-267-1134

E-mail: nashida@ngt.ndu.ac.jp xerostomia (31). Xerostomia arises not only from the systemic disorders, such as Sjögren's disease, but also as a response to various conditions, including adverse drug reactions and stress (9). There are differences in the innervation of the three major salivary glands $(12,25)$, suggesting that the effects of nerve stimulation, drugs, and stress on each salivary gland might be different. Tumor incidence also differs between the salivary glands in humans; the parotid gland has almost a 4-fold higher incidence of tumors than the other glands (16). Recently, differences in gene expression as assessed on DNA microarrays between the human parotid and submandibular glands have been reported (32).

Currently, in cases of salivary gland disease, it is difficult to identify which gland is damaged by analyzing whole saliva because the protein secretion profile of each salivary gland is not well established. Mucin is secreted from both the submandibular and sublingual glands (20), and cystatin is secreted mainly from the submandibular gland (11), but there is no evidence that these proteins are unique to the 
respective salivary glands. Therefore, further analyses are required to determine whether these proteins can be used as specific markers for each salivary gland. It is important to identify the proteins specific to each gland.

In this study, we analyzed the gene expression in rat salivary glands. Rodents are commonly used as animal models for oral biological research; therefore, a basic characterization of the protein profiles of the salivary glands in rats may be useful. We analyzed differential gene expression in the parotid, submandibular, and sublingual glands of rats using DNA microarrays and quantitative real-time polymerase chain reaction (PCR). The purpose of this study was to determine the differences and the similarities in gene expression of the rat major salivary glands, focusing on secretory and secretion-related proteins.

\section{MATERIALS AND METHODS}

RNA isolation. All procedures were conducted according to the Guidelines for the Care and Use of Laboratory Animals, The Nippon Dental University, School of Life Dentistry at Niigata. Male Wistar rats (9 weeks old) were anesthetized with sodium pentobarbital $(50 \mathrm{mg} / \mathrm{kg}$ i.p. $)$ and sacrificed by cardiac puncture. Parotid, submandibular, and sublingual glands were isolated, cut into approximately $30-\mathrm{mg}$ samples, washed with PBS, and soaked in $0.5 \mathrm{~mL}$ RNAlater (Ambion Inc., Austin, TX, USA). Total RNA was prepared using an RNeasy Plus Mini kit (Qiagen, Chatsworth, CA, USA) according to the manufacturer's instructions.

Microarray analysis. Total RNA of parotid, submandibular, and sublingual glands from a single 9-weekold male rat was analyzed with the Whole Rat Genome Oligo Micro array 44K kit (Agilent Technologies Inc., Palo Alto, CA, USA). One-color microarray analysis was performed by Hokkaido System Science Co., Ltd. (Sapporo, Japan). Synthesis of cDNA and the following synthesis of the fluorescent-labeled cRNA were performed from 500-ng samples of total RNA obtained from each of the three salivary glands using the Quick Amp Labeling kit (Agilent Tech). The three fluorescent-labeled cRNA objects were applied to three different arrays on the same slide. The slides were scanned using Agilent Technologies Microarray Scanner. The data were analyzed using GeneSpring software (Agilent Tech).
Real-time quantitative PCR. First-strand cDNA was synthesized from $5 \mu \mathrm{g}$ of total RNA template using a Transcriptor First Strand cDNA Synthesis kit (Roche Diagnostics, Mannheim, Germany) and oligo dT primers according to the manufacturer's instructions. LightCycler (Roche) amplification using a TaqMan probe selected from the Universal ProbeLibrary (Roche) was used for the quantitative real-time PCR assay according to the manufacturer's instruction. Selection of the TaqMan probe and design of the primers listed in Table 1 were performed at the Universal ProbeLibrary Assay Design Center Website. A partial GAPDH cDNA was used as a standard. The concentration of the GAPDH cDNA was determined by measuring the absorbance at $260 \mathrm{~nm}$. The crossing points of unknown samples were determined from the regression line, and the corresponding concentrations were calculated. The transcript copy number was then calculated. The analysis was performed in triplicate using RNA samples from three separate rats.

\section{RESULTS}

Differential gene expression in the three major salivary glands by DNA microarray analysis

To characterize the genes expressed in the three major salivary glands, we performed a DNA microarray analysis. The total gene expression profile of these salivary glands is shown in Fig. 1. All pre-normalized intensity signals from each probe were collated. There were 6211, 7250, and 7505 genes with expression values over 500, for the parotid, submandibular, and sublingual glands, respectively, and these were classified into seven groups as shown in the Venn diagram (Fig. 1a). Group D (genes expressed in the submandibular and sublingual glands) contained a large number of genes (1325 genes), whereas groups B (genes expressed in the parotid and submandibular glands) and $\mathrm{F}$ (genes expressed in the parotid and sublingual glands) contained fewer genes, 53 and 96 genes, respectively.

Gene ontology (GO) analyses were performed to characterize the representation of GO terms of cellular component in groups A, B, C, D, E, F, and G (Fig. 1b). The result showed that all the groups (A-F), except group $\mathrm{G}$, contained the extracellular region GO terms, including the genes related to secretory proteins. All groups that included the parotid gland (groups A, B, and F), except group $\mathrm{G}$, showed a higher ratio of extracellular region $\mathrm{GO}$ terms than the other groups. 
Table 1 Primers used for real-time PCR

\begin{tabular}{|c|c|c|c|c|}
\hline Gene symbol & GenBank accession & Primer & sequence $\left(5^{\prime} \rightarrow 3^{\prime}\right)$ & Amplicon size \\
\hline \multirow[t]{2}{*}{ Amyl } & NM_001010970 & Forward & AGAGGAAACAAAGGCTTCATTG & 74 \\
\hline & & Reverse & GGAAGACCAGTCTGTAGAGTTGTTG & \\
\hline \multirow[t]{2}{*}{ Cst10 } & ENSRNOT00000007336 & Forward & CCTTAGCAGGCCAATACGAG & 61 \\
\hline & & Reverse & AAAGTTCTTCCCAGACACAACC & \\
\hline \multirow[t]{2}{*}{ Gpxl } & NM_030826 & Forward & CGACATCGAACCCGATATAGA & 60 \\
\hline & & Reverse & ATGCCTTAGGGGTTGCTAGG & \\
\hline \multirow[t]{2}{*}{ Muc19 } & XM_235593 & Forward & GATATCCTGGTACCACAGTCACAA & 69 \\
\hline & & Reverse & TCCCATCGGAGCCACTATTA & \\
\hline \multirow[t]{2}{*}{$P s p$} & NM_052808 & Forward & TGCCCCTGGTTTTTGAAAT & 70 \\
\hline & & Reverse & AAGGAAACAGCAACGTCGAG & \\
\hline \multirow[t]{2}{*}{$T f$} & NM_001013110 & Forward & TCCAGAAGGTACCACATATGAAGA & 107 \\
\hline & & Reverse & GGAAAGTGCAGGCTTCTAGG & \\
\hline \multirow[t]{2}{*}{$\mathrm{Ca} 6$} & ENSRNOT00000051309 & Forward & AGGATTCCGCCACCATCT & 61 \\
\hline & & Reverse & TGATCCATTACCGCATTCTCT & \\
\hline \multirow[t]{2}{*}{ Pip } & NM_022708 & Forward & CAATGAAGGCCAGGATAATGA & 75 \\
\hline & & Reverse & TCGTCAGGTGTACTTGGAACA & \\
\hline \multirow[t]{2}{*}{$G A P D H$} & NM_017008 & Forward & TGGGAAGCTGGTCATCAAC & 78 \\
\hline & & Reverse & GCATCACCCCATTTGATGTT & \\
\hline
\end{tabular}

\section{a. Venn diagram}

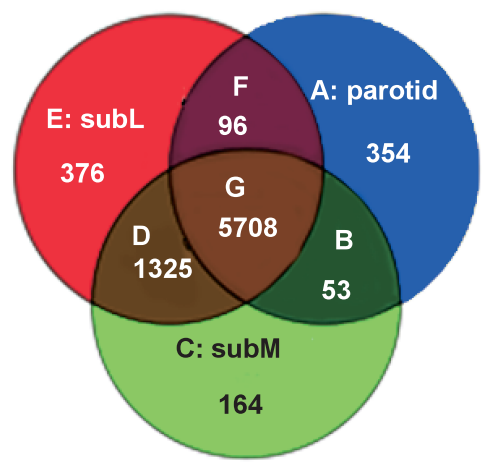

\section{b. GO analysis: Cellular component}

\begin{tabular}{l} 
GO term \\
Extracellular region \\
Cell \\
Membrane-enclosed lumen \\
Envelope \\
Macromolecular complex \\
Organelle \\
Extracellular matrix part \\
Extracellular region part \\
Organelle part \\
Synapse part \\
Cell part \\
Synapse \\
\hline
\end{tabular}

A: parotid

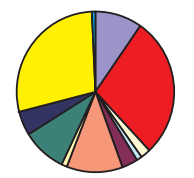

B: parotid\&subM

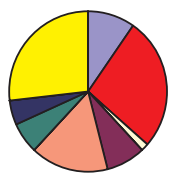

C: subM

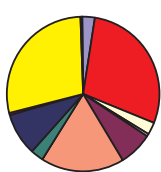

D: subM\&subL

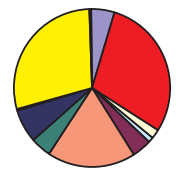

E: subl
$\mathrm{F}$ :

G: subL\&parotid parotid\&subM\&subL

Fig. 1 Gene expression profiles of rat parotid, submandibular, and sublingual glands by DNA microarray. The signals flagging absent and missing values were excluded, and the genes with the expression values over 500 after correction by GAPDH expression were included. A Venn diagram of the genes expressed in the three salivary glands is shown in a. Group A includes 354 genes; group B includes 53 genes; group C includes 164 genes; group D includes 1325 genes; group E includes 376 genes; group F includes 96 genes; group G includes 5708 genes. Cellular component GO analysis on these seven groups classified in $\mathbf{a}$ is shown in $\mathbf{b}$. The ratio of the number of the GO term in the category to the number of the total GO terms in each group is shown as a pie chart. subM: submandibular, subL: sublingual. 
Comparison of gene expression of salivary protein groups in the three major salivary glands

The contribution of the individual salivary glands to the composite salivary protein profile has not been established, although there have been a number of studies on salivary proteins in the whole saliva and in each salivary gland. Therefore, we compared expression among the salivary glands of genes encoding proteins that either might be secreted into saliva or important for secretion.

The expression levels of genes encoding growth factors and mucins, both of which are abundant in human and animal saliva $(20,36)$, are shown in Fig. 2a. It has been reported that the granular duct cells in the mouse submandibular and parotid glands are rich in nerve growth factors (19). In the present study, Ngfg encoding nerve growth factor (NGF) gamma was the most prominently expressed growth factor in the three salivary glands, and its expression level was similar among the three glands. The submandibular and sublingual glands expressed similar, high levels of Muc19, as reported previously (37). The expression of Muc10, which encodes mucin 10-also called submandibular gland mucin (1) - was higher in the parotid gland than in the submandibular and sublingual glands.

The expression of the genes encoding the prolinerich protein (PRP) family is shown in Fig. 2b. The genes encoding PRPs were predominantly expressed in the parotid gland, as reported previously (4), although some genes coding PRPs (e.g., PRP-2, Prp3, and $X M$ 344035, which encodes basic PRP) were expressed in all the three salivary glands at very similar levels.

The expression of genes encoding protease inhibitors, which include the cystatin family, is shown in Fig. 2c. Salivary SD-type cystatins are expressed in serous acini and demilunes in the human salivary glands (11), but only cystatin $\mathrm{S}$ is known to be a saliva-specific cystatin in rats (7). The expression of Cyss, which encodes cystatin $\mathrm{S}$ and has been reported to be augmented by $\beta$-adrenergic stimulation in the rat (11), was lower than the other cystatins in all the three glands. Cystatin C, encoded by Cst3, is a typical cystatin that is distributed to the salivary glands and widely throughout all the organs, as well as cystatin B and cystatin 6. Expression of Cst3, $C s t b$, and Cst6, which encode ubiquitous cystatins, were similar among the three salivary glands in the rat. The highest expression of Cst10, which encodes cystatin 10 (also called carminerin) and contributes to chondrocyte calcification in the mouse (17), was observed predominantly in the parotid gland.
Various proteins showing enzyme activities have been identified in human and animal saliva (10). Genes encoding the salivary enzymes were highly expressed in all salivary glands (Fig. 2d). The genes encoding well-known salivary enzymes, such as amylase, deoxyribonuclease (DNase), carbonic anhydrase VI, lactoperoxidase, and ribonuclease, were predominantly expressed in the parotid gland. Genes encoding the enzymes that are not specific to saliva, such as ribonuclease $\mathrm{P}$ and 6 , arginase, glutathione peroxidase I, and kallikrein, were expressed at similar levels in all three salivary glands.

The expression of the other secretion-related proteins is shown in Fig. 2e. Salivary proteins, including parotid secretory protein (PSP) (15); cysteine-rich secretory protein (encoded by Crisp 1 and Crisp2) (18); and glycoprotein 340 (encoded by $D M B T 1$ ) (24), were expressed at higher levels in the parotid gland than in the other two glands. Albumin (encoded by $A l b), \alpha-2$ macroglobulin (encoded by $A 2 m$ ), $\beta-2$ microglobulin (encoded by $B 2 \mathrm{~m}$ ), and glutathione-S-transferase (encoded by Gspl) are produced mainly in the liver and usually detected in the blood plasma (30), and these genes showed the similar expression levels in the three salivary glands. The submandibular and sublingual glands expressed genes encoding murinoglobulins (Mugl and Mug2) and ovomacroglobulin at higher levels than the parotid gland. The genes encoding calgranulins (S100a8 and S100a9), prolactin-induced protein (Pip), and von Ebner's gland proteins (Vegp1 and Vegp2), which are all detected in human saliva (14), were mainly expressed in the parotid gland.

\section{Differential gene expression by real-time PCR}

To confirm the microarray results, the genes that encode secretory proteins and had distinct expression patterns in the microarray analysis were selected for real-time PCR analysis. The eight genes analyzed by real-time PCR were Muc19, which encodes mucin 19 and was expressed at the high levels both in the submandibular and sublingual glands; Amyl (encoding amylase) and Psp encoding parotid secretory protein, which are observed in abundance in human and rat saliva; Gpxl (encoding glutathione peroxidase 1), the level of which may reflect oxidative stress due to diabetes or drug addiction (2); Tf, which encodes transferrin and is secreted from the rat parotid acinar cells (22); Ca6 (encoding carbonic anhydrase VI); Pip (14); and Cst10. GAPDH expression was used an internal standard, and the expression of each gene was represented relative to $G A P D H$ expression. The results from the real-time 
a

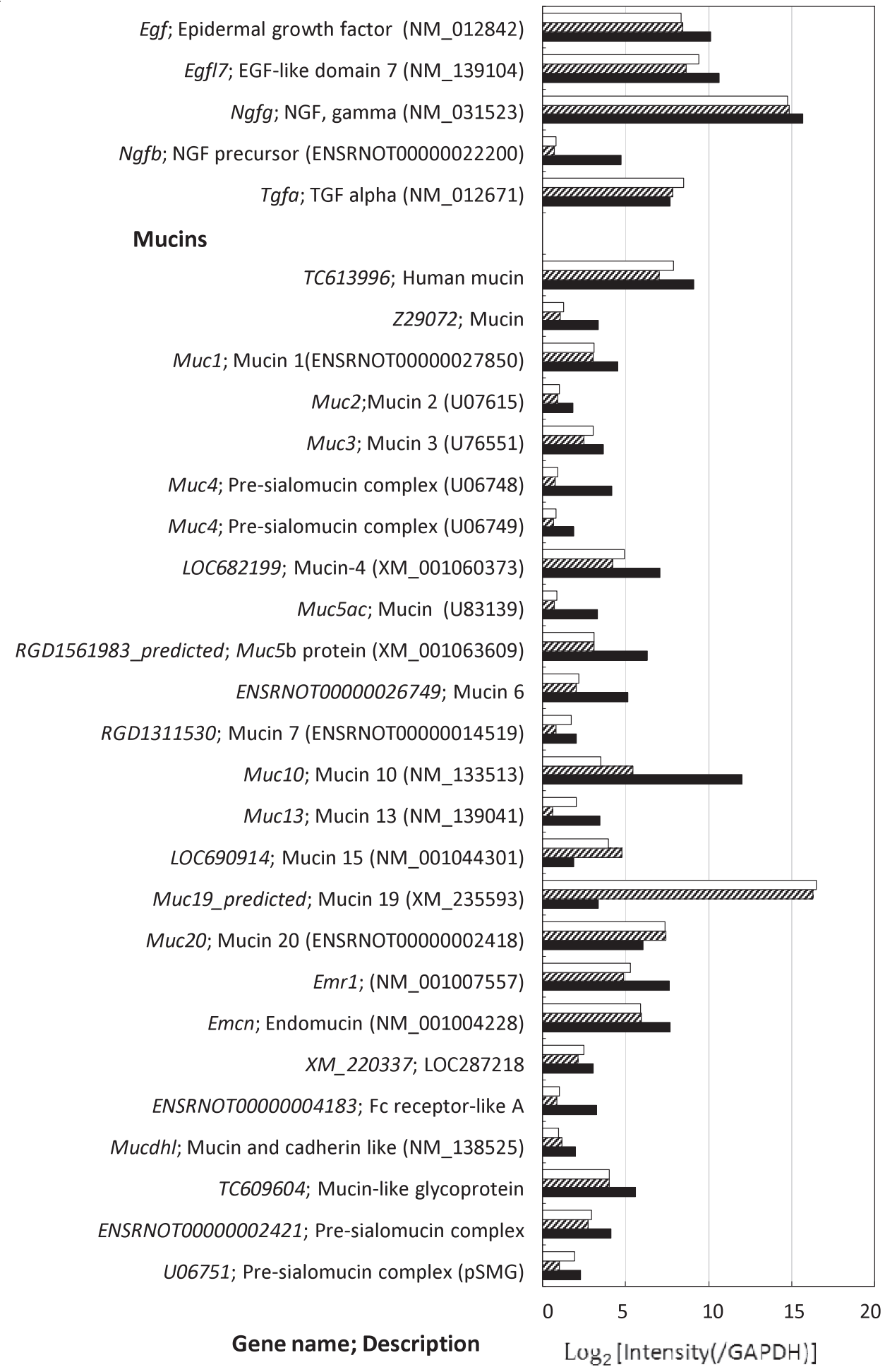

Fig. 2 Comparison of the gene expression in the parotid, submandibular, and sublingual glands on growth factors and mucins (a), proline-rich proteins (b), protease inhibitors (c), enzymes (d), and the other secretion-related proteins (e). Data were indicated as $\log _{2}$ value of the intensity after correction with GAPDH expression. The corrected average of GAPDH was 12.0 . Boolean flags "IsPosAndSignif", which indicate the mark if the mean signal of a feature is greater than the corresponding background and if this difference is significant, were used to pick up genes for the analysis. White columns represent the sublingual gland, striped columns represent the submandibular gland, and black columns represent the parotid gland. 
b

b

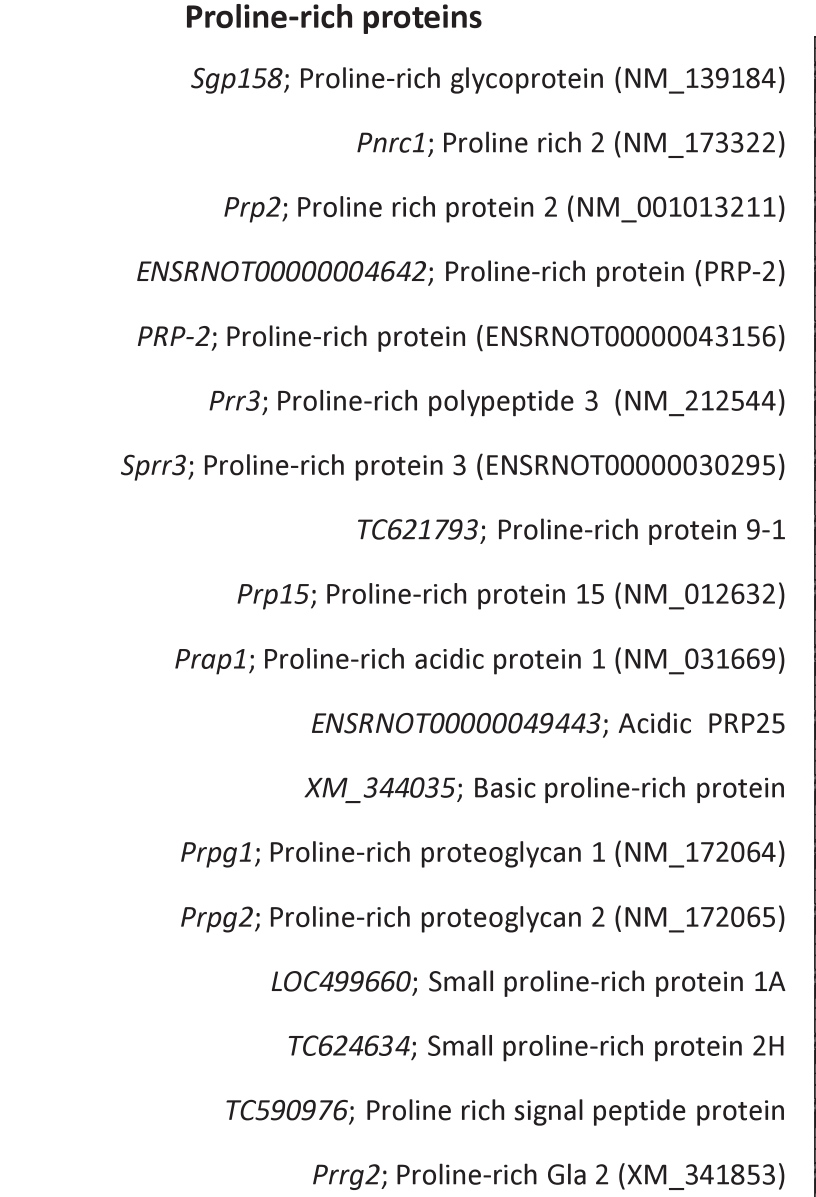

RGD1560542; Proline-rich Gla 4 (ENSRNOT00000038464)

Akt1s1; Proline-rich AKT1 substrate 1 (XM 238103)

Prelp; (NM_053385)

ENSRNOT00000013672; Proline arginine rich coiled coil 1- 5

Kprp; Keratinocytes proline-rich protein (NM_001002290)

Bcl2/12_predicted; BCL2-like 12 (ENSRNOT00000027777)

XM_222968; Hydroxyproline-rich glycoprotein (XM_222968)

gp1 precursor; Hydroxyproline-rich glycoprotein 1 (TC610375)

TC629122; Hydroxyproline-rich glycoprotein-like

\section{Gene name; Description}

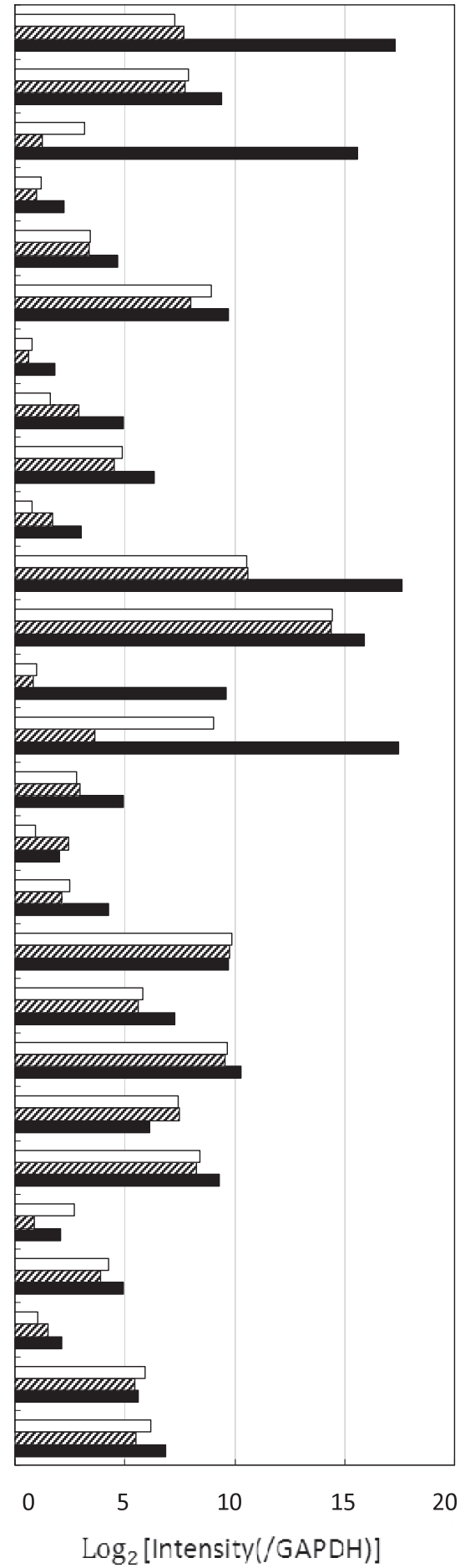


C

Protease inhibitors

Csta_predicted; Cystatin A (ENSRNOT00000039075) Stfa3_predicted; Cystatin alpha (ENSRNOT00000051936) Cstb; Cystatin B (NM_012838) Cst3; Cystatin C (NM_012837) Cst6; Cystatin E/M (Cst6) (NM_133566) ENSRNOT00000006885; Cystatin E2 ENSRNOT00000008865; Cystatin F Cyss; Cystatin S (NM_198685) RGD1566383_predicted; Cystatin S precursor LOC689212; Cystatin S precursor (XM_001069983) LOC257643; Cystatin SC (NM_147137) LOC266776; Cystatin TE-1 (NM_153734) Cst8; Cystatin 8 (NM_019258) Cst9; Cystatin 9 (ENSRNOT00000006869) Cst10; Cystatin 10 (ENSRNOT00000007336) Cst11; Cystatin 11 (NM_139085)

Cst11; Cystatin-like 1 (ENSRNOT00000006362) RGD1563136_predicted; Cystatin-related protein 2 Z13994; Crp2 gene for cystatin related protein 2 Slpi; (NM_053372) RGD1563818_predicted; (XM_215940)

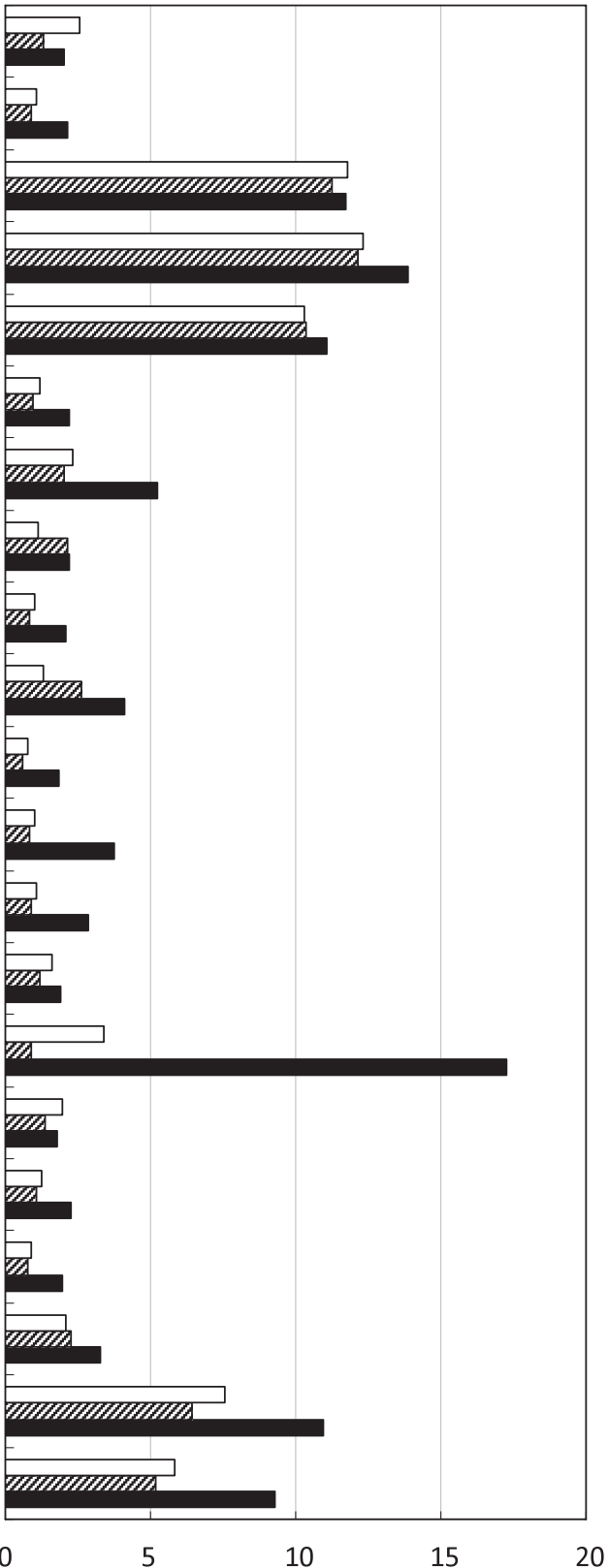

Gene name; Description $\log _{2}[$ Intensity(/GAPDH)] 
d

$$
\begin{array}{r}
\text { Ache; Acetylcholinesterase (X70140) } \\
\text { Alpi2; Alkaline phosphatase (NM_013059) } \\
\text { Alpl; Alkaline phosphatase-II (NM_022680) } \\
\text { Amy1; Amylase } 1 \text { (NM_001010970) } \\
\text { Amy2; Amylase } 2 \text { (NM_031502) } \\
\text { Arg1; Arginase } 1 \text { (NM_017134) } \\
\text { Arg2; Arginase } 2 \text { (NM_019168) }
\end{array}
$$$$
\text { Enzymes }
$$
Bphl; Biphenyl hydrolase-like (NM_001037206) Car1_predicted; Carbonic anhydrase 1

Ca2; Carbonic anhydrase 2 (NM_019291) ENSRNOT00000051309; Carbonic anhydrase 6 Car8; Carbonic anhydrase 8 (NM_001009662)

Car9_predicted; Carbonic anhydrase 9 Dnase1; Deoxyribonuclease I (NM_013097)

G6pdx; G6P dehydrogenase (NM_017006) Gpx1; Glutathione peroxidase 1 (NM_030826)

ENSRNOT00000056448; Kallikrein-3 Lpo_predicted; Lactoperoxidase (ENSRNOT00000011402) TC630388; Lactoperoxidase Lyz; Lysozyme (NM_012771)

TC640203; Lysozyme LOC688047; Lysozyme C2 (XM_001080970) LyzI1_predicted; Lysozyme-like 1 Lyz/4_predicted; Lysozyme-like 4 Lyzl6_predicted; Lysozyme-like 6 (XM_213497) ENSRNOTO0000052339; Maltase Rnaset2_predicted; Ribonuclease 6 Rnase1_predicted; Ribonuclease A 1 (NM_001013232) Rnase1; Ribonuclease A1 (NM_001029904) TC614297; Ribonuclease alpha (TC614297) NP516904; Ribonuclease beta (NP516904) NP516897; Ribonuclease gamma (NP516897) Pop5_predicted; Ribonuclease P Rpp21; Ribonuclease P 21 (NM_001002831) Pop7_predicted; Ribonuclease P family (XM_213733)

\section{Gene name; Description}

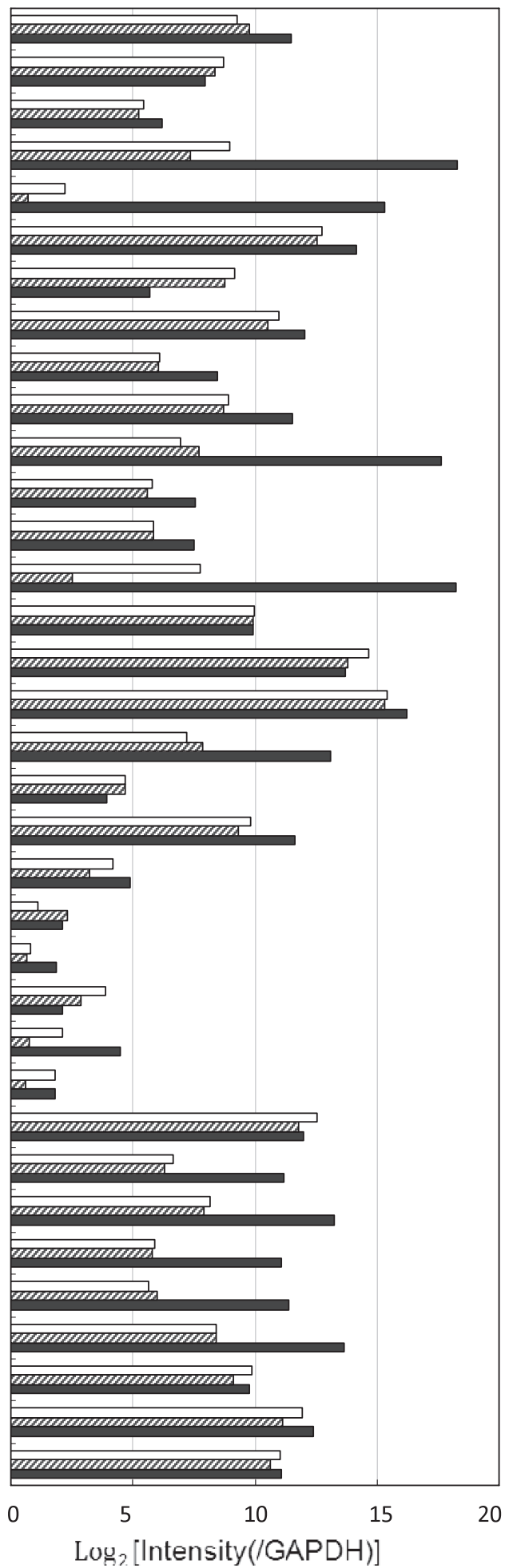


e

Other proteins

Alb; Albumin (NM_134326)

Azgp1; Alpha-2-glycoprotein 1 (NM_012826)

A2m; Alpha-2-macroglobulin (NM_012488)

Lrpap1; Alpha-2-macroglobulin receptor-associated protein

B2m; Beta-2 microglobulin (NM_012512)

S100a8; Calgranulin A (NM_053822)

S100a9; Calgranulin B (NM_053587)

Crispld1_predicted; (ENSRNOT00000024199)

Crispld2; (NM_138518)

Crisp1; Cysteine-rich secretory protein 1 (NM_022859)

Crisp2; Cysteine-rich secretory protein 2 (NM_031240)

Dmbt1; (XM_577842)

Fabp5; Fatty acid binding protein 5 (NM_145878)

Gstp1; Glutathione-S-transferase (NM_012577)

Hrg; Histidine-rich glycoprotein (NM_133428)

IIrap; (NM_012968)

Ltf_predicted; Lactotransferrin (ENSRNOT00000056066)

ENSRNOT00000046109; Lipocalin-4

Mug1; Murinoglobulin 1 (NM_023103)

Mug2; Murinoglobulin 2 (NM_001002826)

Orm1; Orosomucoid 1(NM_053288)

RGD1565709_predicted; Ovomacroglobulin (XM_342764)

Psp; Parotid secretory protein (NM_052808)

Pnsp1; (NM_001024283)

Pip; Prolactin induced protein (NM_022708)

ENSRNOTO0000011906; Proline rich membrane anchor 1

Sec14/3; 45 kDa Secretory protein (AJ132352)

Sgne1; (NM_013175)

Chi3/3; Secretory protein (XM_227566)

Tgfb1i4; TGF beta 1 induced transcript 4 (BC059146)

Tf; Transferrin (NM_001013110)

Vegp1; Von Ebners gland protein 1 (NM_022945)

Vegp2; Von Ebners gland protein 2 (NM_053574)

LOC338400; Von Ebner's-like protein (AF123454)

\section{Gene name; Description}

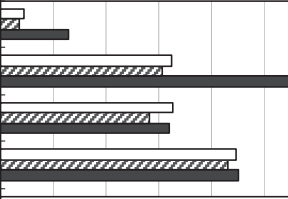

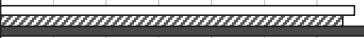

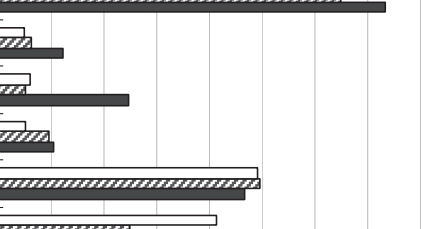

200002000

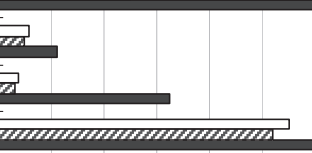

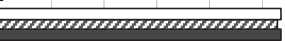

202

200002000000000

觖

a

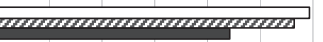

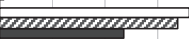

a

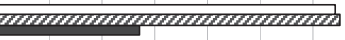

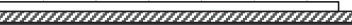

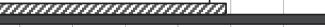

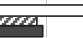

2000020000000

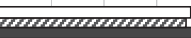

202

ZI

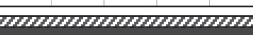

(2)

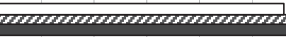

$\infty$

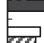

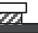

8

16

20

$\log _{2}[$ Intensity (/GAPDH)] 
PCR were compared with the results from the DNA microarray analysis of one rat (Fig. 3). Relative expression of Cst10, Amyl, Psp, Tf, Ca6, and Pip was higher in the parotid gland than in the submandibular and sublingual glands, whereas Muc19 was specifically expressed in the submandibular and sublingual glands, and the expression of Gpxl was apparently higher in the submandibular glands than in the other glands. The results from the real-time PCR were, in the aggregate, consistent with the results from the DNA microarray analysis, although the relative expression levels were not the same between the results from the two methods.

\section{DISCUSSION}

There are a number of studies on the expression and distribution of proteins in the parotid, submandibular, and sublingual glands $(4,10,11,14,20,24)$. However, there are few reports that simultaneously compare gene expression in the three major salivary glands, although the gene expression of human parotid and submandibular glands has been compared for tumor incidence in a previous study (32). In this investigation, we analyzed gene expression in the rat salivary glands using DNA microarrays and assessed the microarray results using real-time PCR. The results on eight genes from the real-time PCR were largely consistent with the results from the microarray analysis (Fig. 3); therefore, the results on the large number of genes in the DNA microarray analysis may be useful in comparing gene expression in the three salivary glands.

It has been reported that the submandibular gland is a mixed gland with serous and mucous acini, the sublingual gland contains mainly mucous acini, and the parotid gland is composed of only serous acini (13). Our results suggested that the gene expression profiles of the submandibular and sublingual glands were very similar and that the profile of the parotid gland was considerably different from the profiles of the submandibular and sublingual glands (Fig. 2).

Salivary mucins are the component that make saliva viscous and are generally known to be secreted from the submandibular and sublingual glands. Our investigation revealed that large numbers of mucin genes were also highly expressed in the parotid gland, although only Muc19 (encoding mucin 19), which showed the highest expression among the mucins investigated, was much more highly expressed in both the submandibular and sublingual glands than in the parotid gland. Mucins encoded by MUC2, MUC5AC, MUC5B, MUC6, and MUC7 are known as the secreted mucin in human saliva. The genes of mucin $5 \mathrm{ac}$ and $5 \mathrm{~b}$ (Muc5ac and Muc5b), as well as $M u c 6$, were expressed in all three rat salivary glands, but mainly in the parotid gland. These results indicated that a number of mucins may be expressed not only in the mucous cells but also in the serous cells. Muc10 was expressed in the rat parotid gland, whereas the protein mucin 10 has not been detected in human saliva as well as mucin 19 (1). In the rodent salivary glands, Muc10 may be expressed specifically in the parotid gland, and Muc19 specifically in the submandibular and sublingual glands.

It has been reported that saliva contains various growth factors (36) and that the submandibular gland secretes NGF and transforming growth factor (TGF) $\beta$ (28). In our results, all three salivary glands expressed the genes encoding TGF, epidermal growth factor (EGF), and NGF, and the expression of these genes was highest in the parotid gland. The submandibular gland is the largest salivary gland in rodents and humans; therefore, it may produce and secrete the largest amount of growth factors.

It has been reported that PRPs are secreted from the parotid gland (4). However, our results revealed that the submandibular and sublingual glands also expressed the basic PRP gene and several other types of PRP genes, indicating that PRPs are not specific to the parotid gland. Proline-rich proteoglycans (6) were much more highly expressed in the parotid gland than the other glands, and their expression may be specific to the parotid gland.

The ubiquitous cystatins, Cstb, Cst 3 , and Cst6 (encoding cystatin $\mathrm{E} / \mathrm{M}$ ), were expressed in all the three glands at very similar levels, consistent with previous results (11). Cst10 which has been reported in developing cartilage of the mouse but not in humans (17) was expressed at the high levels mainly in the rat parotid gland. This is the first report showing Cst10 expression in the soft tissues, but the expression and the role of carminerin encoded by Cst10 in the parotid gland is unknown. The gene Cyss, which encodes cysteine $\mathrm{S}$ and has been reported to be produced in response to $\beta$-adrenergic stimulation (7), was expressed at low levels in all three glands. Further examination is required to determine in which gland(s) Cyss is up-regulated in response to $\beta$-adrenergic stimulation.

The parotid gland contributed to the expression of almost all the genes encoding salivary-specific enzymes that were investigated. Specifically, the genes encoding amylase, DNase, and carbonic anhydrase 

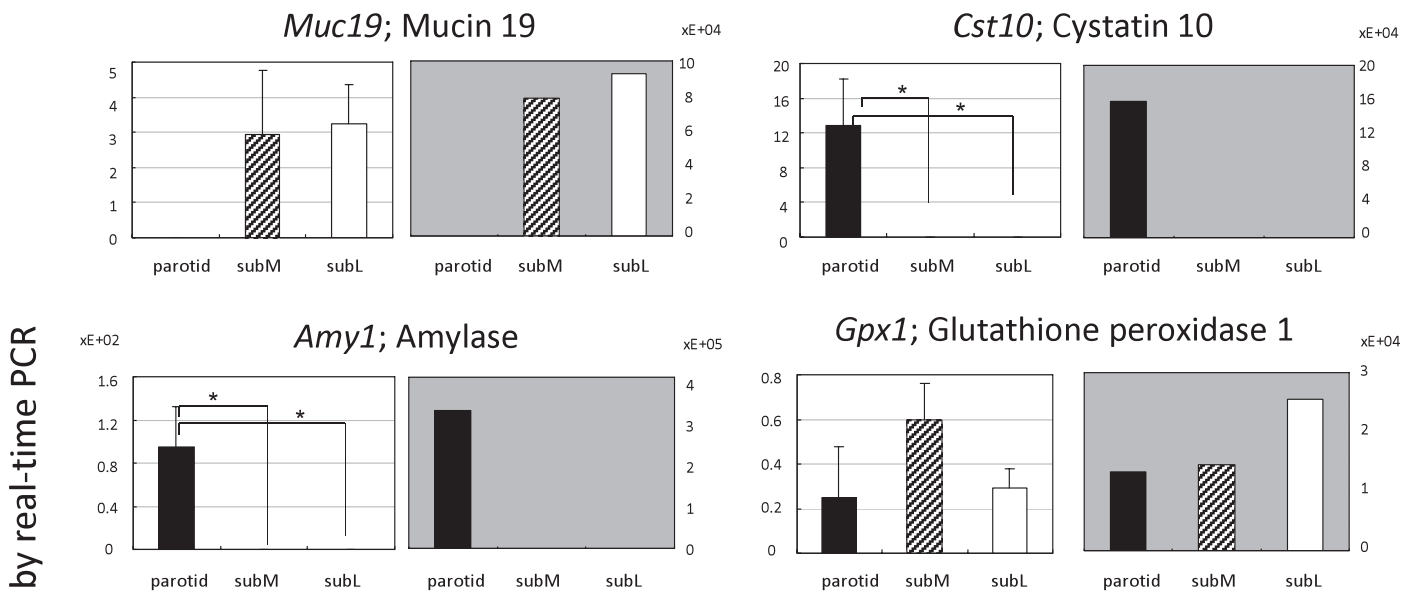

Fig. 3 Comparison of gene expression of secretory and secretion-related proteins among the parotid (black columns), submandibular (striped columns), and sublingual glands (white columns) of rats by real-time PCR analysis (right) and DNA microarray (left). The bar graphs represent expression relative to the GAPDH standard. Statistical analyses of the differences between the relative expressions in the salivary glands were evaluated by ANOVA. $\left(n=3\right.$, for the real-time $P C R ;{ }^{\star} P<0.05$; $\left.{ }^{* *} P<0.01\right)$.

VI were expressed in the parotid gland, while the genes encoding lactoperoxidase, glutathione peroxidase, kallikrein, and lysozyme (none of which are salivary specific) were expressed at similar levels in all the three glands, including the parotid gland. It is well-known that amylase and carbonic anhydrase VI are mainly secreted from the serous cells of the parotid glands, but the origins of the other enzymes have not been established. Further investigation of the expression of these enzymes is required.

Genes encoding proteins that could not be classified into any of the categories described above were also investigated. The gene encoding ovomacroglobulin, which is an $\alpha 2$-macroglobulin-like protease inhibitor, was expressed in the submandibular and sublingual glands. Crisp1 encoding a member of the cysteine-rich secretory proteins (CRISPs), which may play a role in the innate immune system (18), and $P S P$, which encodes a bacteria-agglutinating peptide (15), were mainly expressed in the parotid gland. The genes encoding some blood proteins were expressed at similar levels in all the salivary glands. Specifically, the genes encoding $\beta 2$-microglobulin, which is reported to occur at higher density in saliva of patients with Sjögren's syndrome (21); $\alpha 2$ macroglobulin, which is a protease inhibitor (3); and transferrin, which is an iron-binding protein, were highly expressed in all three glands. These proteins may not originate from blood but rather produced locally in the salivary glands. Further investigation for examination of this possibility is required. $L f n$ coding lactoferrin was expressed only at low levels 
in rat salivary glands, which is interesting given that it has an antibacterial effect in the oral cavity of human (33).

Our investigation revealed that a large number of genes encoding secretory proteins were expressed mainly in the parotid gland and that some secretory proteins were highly expressed in both the submandibular and sublingual glands with low parotid gland expression. Our results suggest that the analysis of salivary proteins may be useful in distinguishing the disorders of the parotid gland from those of the submandibular and sublingual glands. In contrast, analysis of salivary proteins may not be useful in distinguishing the disorders of the submandibular gland from those of the sublingual glands.

The investigation of proteins in human saliva (14) demonstrates that many proteins encoded by the genes analyzed in our investigation are also detected in human saliva; therefore, these proteins may be useful as markers for the different salivary glands in humans. However, there are some genes which are highly expressed but are known not to be translated into proteins. For example, the expression of mucin 19 has not been confirmed in human saliva, whereas the encoding gene Muc19 has been detected in the human submandibular gland (8). Detection of genes in saliva may be useful in assigning specific transcripts to each salivary gland rather than specific proteins. Further examination will be required to determine whether the gene expression profiles of the human salivary glands correspond to the appearance of proteins in human saliva.

\section{Acknowledgment}

This work was supported by a Grant-in-Aid for Scientific Research from JSPS (20592380).

\section{REFERENCES}

1. Albone EF, Hagen FK, Van Wuyckhuyse BC and Tabak LA (1994) Molecular cloning of a rat submandibular gland apomucin. J Biol Chem 269, 16845-16852.

2. Arana C, Cutando A, Ferrera MJ, Gomez-Moreno G, Worf CV, Bolanos MJ, Escames G and Acuna-Castroviejo D (2006) Parameters of oxidative stress in saliva from diabetic and parenteral drug addict patients. J Oral Pathol Med 35, 554559.

3. Arnold JN, Wallis R, Willis AC, Harvey DJ, Royle L, Dwek RA, Rudd PM and Sim RB (2006) Interaction of mannan binding lectin with alpha2 macroglobulin via exposed oligomannose glycans: a conserved feature of the thiol ester protein family? J Biol Chem 281, 6955-6963.

4. Carlson DM (1993) Salivary proline-rich proteins: biochemistry, molecular biology, and regulation of expression. Crit Rev Oral Biol Med 4, 495-502.
5. Carpenter GH, Proctor GB, Pankhurst CL, O'donohue J, Scotto D and Hunnable MP (2000) Sialochemical markers of salivary gland involvement with Sjögren's syndrome secondary to rheumatoid arthritis and primary biliary cirrhosis. $J$ Oral Pathol Med 29, 452-459.

6. Castle AM and Castle JD (1993) Novel secretory proline-rich proteoglycans from rat parotid. Cloning and characterization by expression in AtT-20 cells. $J$ Biol Chem 268, 2049020496.

7. Chaparro O, Yu WH and Shaw PA (1997) Effect of sympathectomy on isoproterenol-induced expression of the cysteine proteinase inhibitor gene, cystatin $\mathrm{S}$, in rat submandibular glands. Arch Oral Biol 42, 743-754.

8. Chen Y, Zhao YH, Kalaslavadi TB, Hamati E, Nehrke K, Le AD, Ann DK and Wu R (2004) Genome-wide search and identification of a novel gel-forming mucin MUC19/Muc19 in glandular tissues. Am J Respir Cell Mol Biol 30, 155-165.

9. Daniels TE and Wu AJ (2000) Xerostomia-clinical evaluation and treatment in general practice. $J$ Calif Dent Assoc 28, 933-941.

10. Denny P, Hagen FK, Hardt M, Liao L, Yan W, Arellanno M, Bassilian S, Bedi GS, Boontheung P, Cociorva D, Delahunty CM, Denny T, Dunsmore J, Faull KF, Gilligan J, GonzalezBegne M, Halgand F, Hall SC, Han X, Henson B, Hewel J, $\mathrm{Hu}$ S, Jeffrey S, Jiang J, Loo JA, Ogorzalek Loo RR, Malamud D, Melvin JE, Miroshnychenko O, Navazesh M, Niles R, Park SK, Prakobphol A, Ramachandran P, RIchert M, Robinson S, Sondej M, Souda P, Sullivan MA, Takashima J, Than S, Wang J, Whitelegge JP, Witkowska HE, Wolinsky L, Xie Y, Xu T, Yu W, Ytterberg J, Wong DT, Yates Jr 3rd and Fisher SJ (2008) The proteomes of human parotid and submandibular/sublingual gland salivas collected as the ductal secretions. J Proteome Res 7, 1994-2006.

11. Dickinson DP (2002) Salivary (SD-type) cystatins: over one billion years in the making - but to what purpose? Crit Rev Oral Biol Med 13, 485-508.

12. Emmelin N (1987) Nerve interactions in salivary glands. $J$ Dent Res 66, 509-517.

13. Eversole LR (1972) The mucoprotein histochemistry of human mucous acinar cell containing salivary glands: submandibular and sublingual glands. Arch Oral Biol 17, 43-53.

14. Ghafouri B, Tagesson C and Lindahl M (2003) Mapping of proteins in human saliva using two-dimensional gel electrophoresis and peptide mass fingerprinting. Proteomics 3, 1003-1015.

15. Gorr SU, Sotsky JB, Shelar AP and Demuth DR (2008) Design of bacteria-agglutinating peptides derived from parotid secretory protein, a member of the bactericidal/permeability increasing-like protein family. Peptides 29, 2118-2127.

16. Ito FA, Ito K, Vargas PA, De almeida OP and Lopes MA (2005) Salivary gland tumors in a Brazilian population: a retrospective study of 496 cases. Int J Oral Maxillofac Surg 34, 533-536.

17. Koshizuka Y, Yamada T, Hoshi K, Ogasawara T, Chung UI, Kawano H, Nakamura Y, Nakamura K, Ikegawa S and Kawaguchi H (2003) Cystatin 10, a novel chondrocytespecific protein, may promote the last steps of the chondrocyte differentiation pathway. J Biol Chem 278, 48259-48266.

18. Kratzschmar J, Haendler B, Eberspaecher U, Roosterman D, Donner P and Schleuning WD (1996) The human cysteinerich secretory protein (CRISP) family. Primary structure and tissue distribution of CRISP-1, CRISP-2 and CRISP-3. Eur $J$ Biochem 236, 827-836.

19. Kurabuchi S and Hosoi K (2009) Immunocytochemical study 
of granular duct cells with a hormonally enhanced granular cell phenotype in the mouse parotid gland. Odontology 97, $57-61$.

20. Levine MJ, Reddy MS, Tabak LA, Loomis RE, Bergey EJ, Jones PC, Cohen RE, Stinson MW and Al-Hashimi I (1987) Structural aspects of salivary glycoproteins. J Dent Res 66, 436-441.

21. Mathews SA, Kurien BT and Scofield RH (2008) Oral manifestations of Sjögren's syndrome. J Dent Res 87, 308-318.

22. Mestecky J (1993) Saliva as a manifestation of the common mucosal immune system. Ann N Y Acad Sci 694, 184-94.

23. Nashida T, Yoshie S, Imai A and Shimomura H (2009) Transferrin secretory pathways in rat parotid acinar cells. Arch Biochem Biophys 487, 131-138.

24. Prakobphol A, Xu F, Hoang VM, Larsson T, Bergstrom J, Jophansson I, Frangsmyr L, Holmskov U, Leffler H, Nilsson C, Boren T, Wright JR, Stromberg N and Fisher SJ (2000) Salivary agglutinin, which binds Streptococcus mutans and Helicobacter pylori, is the lung scavenger receptor cysteinerich protein gp340. J Biol Chem 275, 39860-39866.

25. Proctor GB and Carpenter GH (2007) Regulation of salivary gland function by autonomic nerves. Auton Neurosci 133, 318.

26. Roescher N, Tak P and Illei G (2009) Cytokines in Sjögren's syndrome. Oral Dis 15, 519-526.

27. Rousseau K, Kirkham S, Johnson L, Fitzpatrick B, Howard M, Adams EJ, Rogers DF, Knight D, Clegg P and Thornton DJ (2008) Proteomic analysis of polymeric salivary mucins: no evidence for MUC19 in human saliva. Biochem J 413, 54552 .

28. Sabbadini E and Berczi I (1995) The submandibular gland: a key organ in the neuro-immuno-regulatory network? Neuro- immunomodulation 2, 184-202.

29. Schneyer LH and Levin LK (1955) Rate of secretion by individual salivary gland pairs of man under conditions of reduced exogenous stimulation. $J$ Appl Physiol 7, 508-512.

30. Schwick HG and Haupt H (1981) Purified human plasma proteins of unknown function. Jpn J Med Sci Biol 34, 299327.

31. Sreebny LM (2000) Saliva in health and disease: an appraisal and update. Int Dent $J$ 50, 140-161.

32. Sun QF, Sun QH, Du J and Wang S (2008) Differential gene expression profiles of normal human parotid and submandibular glands. Oral Dis 14, 500-509.

33. Weinberg ED (2001) Human lactoferrin: a novel therapeutic with broad spectrum potential. J Pharm Pharmacol 53, 1303-1310.

34. Yavuz S, Toker E, Bicakcigil M, Mumcu G and Cakir S (2006) Comparative analysis of autoantibodies against $\alpha$-fodrin in serum, tear fluid, and saliva from patients with Sjögren's syndrome. J Rheumatol 33, 1289-1292.

35. Yu DF, Chen Y, Han JM, Zhang H, Chen XP, Zou WJ, Liang LY, Xu CC and Liu ZG (2008) MUC19 expression in human ocular surface and lacrimal gland and its alteration in Sjögren syndrome patients. Exp Eye Res 86, 403-411.

36. Zelles T, Purushotham KR, Macauley SP, Oxford GE and Humphreys-Beher MG (1995) Saliva and growth factors: the fountain of youth resides in us all. J Dent Res 74, 18261832.

37. Zinzen KM, Hand AR, Yankova M, Ball WD and Mirels L (2004) Molecular cloning and characterization of the neonatal rat and mouse submandibular gland protein SMGC. Gene 334, 23-33. 